\title{
Effect of Sleep State on the QT Interval in Normal Infants
}

\author{
GABRIEL G. HADDAD, ${ }^{28}$ EHUD KRONGRAD, RALPH A. EPSTEIN, MARY ANNE F. EPSTEIN, \\ H. STEPHEN LAW, JUDY B. KATZ, NORMAN M. MAZZA, AND ROBERT B. MELLINS
}

Departments of Pediatrics, Anesthesiology, and Chemical Engineering and Applied Chemistry, Columbia University and Babies Hospital, New York, USA

\begin{abstract}
Summary
Twenty QT intervals selected at random from the middle periods of rapid eye movement (REM) and quiet sleep were measured in 12 normal infants studied at 2 weeks and 1, 2,3, and 4 months of life. A digitizing system, consisting of a precision rotational potentiometer mounted on a pair of calipers and an $A / D$ converter, was used for measurements. An accuracy of \pm 2 msec was achieved by high resolution of the digitized signal and calibration of each QT measurement with an accurately generated time code. Sleep staging was done visually using an electroencephalogram (EEG), an electrooculogram (EOG), a submental electromyogram (EMG), and behavioral criteria. Our results show that the QT index $\left(\mathbf{Q T}_{c}\right.$ $=\mathbf{Q T} / \sqrt{\mathbf{R R}}$ ) was significantly greater during quiet sleep (mean $=0.439)$ than during REM sleep (mean $=0.433)(P<0.01)$ and that this difference existed at all ages studied.
\end{abstract}

\section{Speculation}

Sleep states are shown to have detectable effects on the duration of ventricular repolarization as measured by the $Q T$ interval. We speculate that this sleep state-related difference in the QT interval may be caused by an increased sympathetic activity in REM sleep.

Prolongation of the QT interval has been implicated in the genesis of ventricular fibrillation and sudden death $(17,20,24)$. Such prolongation of the QT interval has been postulated to occur in REM sleep (20), which occupies a greater proportion of sleep in very young infants. This might, therefore, predispose to sudden death during sleep (sudden infant death syndrome or SIDS). Before being able to evaluate the QT interval during sleep in the infant at risk for SIDS, it is necessary to examine the effect of sleep state on the QT interval in normal infants. In this study, we measured the QT interval in both quiet and REM sleep in order to determine the relation of the sleep state to the repolarization process of the ventricles in normal infants during the first 4 months of life.

\section{MATERIALS AND METHODS}

\section{PATIENT POPULATION}

Thirty-seven studies were performed in 12 normal infants during sleep. Pregnancy, delivery, and neonatal course were uneventful. Birth weight ranged from 2900 to $4560 \mathrm{~g}$ and gestational age from 38 to 42 weeks. There were seven males and five females. Written informed consent was obtained from the parents of all infants. Infants were studied for 2 to $3 \mathrm{hr}$ after the midmorning feed at 2 weeks and 1, 2, 3 and 4 months of age. Ten infants were studied three to five times; two infants were studied only once.

None of the infants was on any medication and none had any clinical evidence of electrolyte imbalance. There was no history of SIDS in their siblings nor was there a family history of deafness, an abnormality that has been associated in many instances with prolongation of the QT interval (14). Eight infants are now over 1 year old; all have normal neurologic and developmental examinations. Conventional 12-lead electrocardiograms performed at the time of each study were normal in all infants.

\section{NEUROPHYSIOLOGIC AND ELECTROCARDIOGRAPHIC SIGNALS}

Standard surface electrodes were used to record the following signals: 1) C4-Al EEG, 2) single bipolar EOG, 3) submental EMG, and 4) bipolar chest electrocardiogram. The electrocardiogram (ECG) electrodes were placed on the anterior chest, one to the left of the manubrium and the other 1 to $5 \mathrm{~cm}$ inferiorly and to the left of the first electrode. The electrode position was not changed during a study. There was no detectable electrical noise on any signal. All signals, including an infant behavioral code, were recorded on a polygraph. The behavioral code included such somatic activities as movements of the extremities, facial grimaces and sucking, total body movements, and whether the eyes were open or closed. The frequency response of the ECG system was 0.05 to $200 \mathrm{~Hz}$. The raw data (EEG, EOG, EMG, ECG, and behavioral code) and a computer-generated time code with an accuracy of $0.02 \%$ were recorded simultaneously on analog magnetic tape (IRIG intermediate band, frequency response 0-312 $\mathrm{Hz}$ ) for future display and analysis. Sleep staging was visually performed on 30-sec epochs by two independent observers using the EEG, EOG, EMG, and behavioral code (3).

\section{MEASUREMENT OF QT INTERVAL DURING SLEEP}

To measure and analyze the QT interval, short periods of 1-2 min of the ECG and time code signals were chosen from the middle of REM and quiet sleep periods for playback onto the polygraph which was run at $100 \mathrm{~mm} / \mathrm{sec}$. The tape recording and playback speeds were the same. Twenty QT intervals were selected from each of these periods for measurement using tables of random numbers (9). Approximately 2000 QT intervals and their corresponding RR intervals were measured. Only studies in which the end of the $T$ wave could be clearly defined were included in this series.

Since studies in newborn and young infants have shown that the heart rate decreases as a function of time during sleep (19), all QT and RR measurements were chosen from the middle of each stage during the first sleep cycle to allow consistent sampling. The QT intervals in REM sleep were selected to coincide with periods of actual rapid eye movements.

A digitizing system, consisting of a precision rotational potentiometer (Computer Instruments Corp., New York, NY) mounted on a pair of calipers, and an A/D converter, was used for these measurements. For maximal precision, each measurement was sampled 100 times and averaged by a minicomputer. To avoid dependence on the accuracy of the tape recorder and the polygraph drives, the electronically generated $1-\mathrm{sec}$ interval was recorded continuously and used for calibration of each QT and RR 


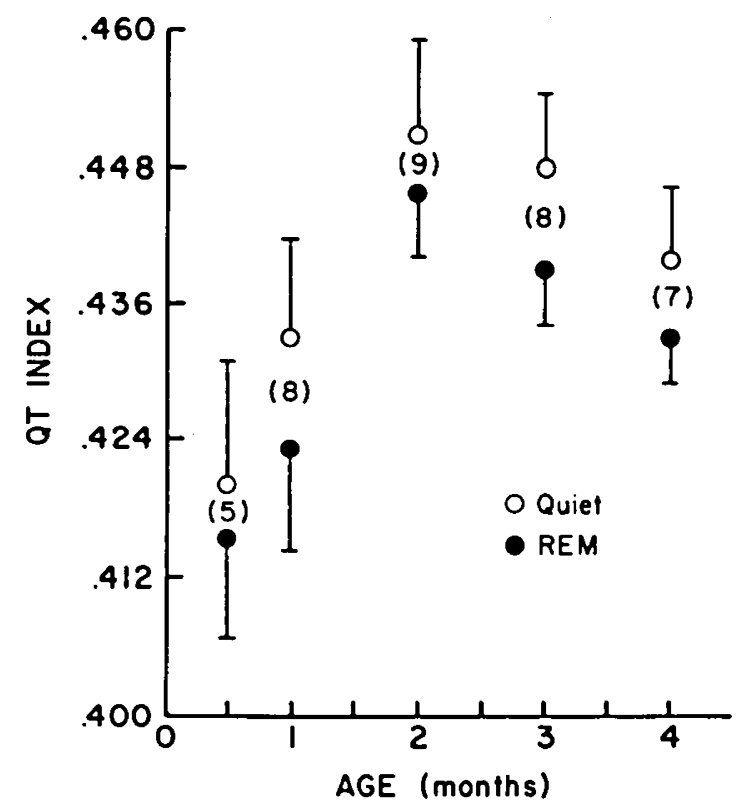

Fig. 1. QT index as a function of age during REM and quiet sleep for 12 normal infants. Mean and 1 SD are shown. The number of infants studied at each age is in parenthesis. Statistically significant differences between the means were present at 1,3 , and 4 months $(P<0.05)$.

measurement. The end of the $\mathrm{T}$ wave was determined by placing a straight edge on the isoelectric line and another on the final slope of the $\mathbf{T}$ wave. Error analysis of our technique disclosed a total potential error of $2 \mathrm{msec}$ or less. Although the nonlinearity of the potentiometer was eliminated by softwave linearization, the potential errors were mainly due to the $A / D$ conversion and the frequency response of the polygraph. There was no measurable distortion of the ECG signal by the taping system.

The heart rate corrected QT interval or QT index $\left(\mathrm{QT}_{\mathrm{c}}\right)$ was calculated using Bazett's formula (6), $\mathrm{QT}_{\mathrm{c}}=\mathrm{QT} / \sqrt{\mathrm{RR}}$, and is reported as a dimensionless value. The mean of $20 \mathrm{QT}$ indices was determined for each sleep state in each study. Paired $t$ test and sign tests were used for statistical analysis.

\section{RESULTS}

The QT index during the first 4 months of life ranged from $0.384-0.466$ in REM sleep and from $0.391-0.479$ in quiet sleep. In 27 of 37 studies, the QT index was greater in quiet than in REM sleep $(P<0.01$, sign test). Although the mean difference was not large, the paired $t$ test for the data taken as a whole confirmed that the QT index in REM (mean $=0.433, \mathrm{SD}=0.017$ ) was smaller than the QT index in quiet sleep (mean $=0.439, \mathrm{SD}=$ $0.019)(P<0.01)$. Despite the relatively small number of subjects, the difference existed at all ages studied and was significant at 1 , 3 , and 4 months of age $(P<0.05$, paired $t$ test) (Fig. 1). Since the placement of the ECG electrodes on the chest varied from study to study and the axis of the heart changes with age, comparison of absolute $\mathrm{QT}_{\mathrm{c}}$ values at different ages could not be done.

\section{DISCUSSION}

The present study demonstrates that the QT index is greater in quiet than in REM sleep in normal infants. The wider frequency response of our equipment along with the faster paper speed, accurate time reference code, and computer-assisted averaging procedure allowed us to evaluate the QT interval with more accuracy than is possible by conventional methods. The small difference we observed between quiet and REM sleep may not be detectable with conventional equipment. However, all of the values we found in sleeping infants fall within the wide range generally accepted as normal $(2,18,25)$. Although Harper et al.
(12) failed to demonstrate an effect of sleep state on QT interval, the results of these investigations may not be comparable with ours since they measured the time interval between the onset of the QRS and the peak of the $T$ wave rather than to the end of the $T$ wave.

Correction of the QT interval for heart rate using Bazett's formula may not be ideal, since it assumes that the relationship between the QT and RR intervals remains the same throughout the entire range of $R R$ intervals. Although more recent studies have led to the development of better-fitting functions (23), Bazett's method of correcting the QT interval for heart rate is adequate when the RR intervals are in a range similar to ours and when differences in heart rate between the two states is small (23). Investigation of the QT-RR relation in our data showed a linear dependence of the QT on the RR interval in some studies and an absence of such dependence in others. In the latter group, the range of the RR interval was too small $(30-50 \mathrm{msec})$ for the QT interval to show any clear dependence. These and other studies from this laboratory (16) demonstrate that the RR interval is shorter in REM than in quiet sleep. Therefore, the use of Bazett's or other methods (23) would tend to minimize the observed difference in the $\mathrm{QT}_{\mathrm{c}}$ between REM and quiet sleep.

In the absence of electrolyte disturbances or drug intake, the QT index varies with the activity of the autonomic nervous system $(10,11,15,22)$, which is known to vary with the sleep state $(4,5$, 13). Experimental studies have indicated that REM sleep, in the presence of actual eye movements, is characterized by an increase in sympathetic activity while quiet sleep is characterized by an increase in parasympathetic activity $(4,5,13)$. We believe that the differences in QT index in REM and quiet sleep reflect differences in the relative activity of the sympathetic and parasympathetic nervous systems in the two sleep states.

Although there are no direct experimental data on the duration of ventricular repolarization during the various sleep states, indirect evidence suggests that the autonomic nervous system regulates the duration of repolarization during sleep. Several investigators have found that bilateral surgical ablation of the cardiac sympathetic nerves results in an increase in the refractory period $(11,21$, 22). Epinephrine and isoproterenol increase the negative slope of the second phase of repolarization and decrease the plateau time of the action potential increasing the rate of repolarization in isolated Purkinje fibers; $\beta$ blockade inhibits the effect of these sympathomimetic agents (10). Continuously infused catecholamines in vivo also shorten the QT interval (1). Although the duration of repolarization and the duration of the QT interval are not always correlated, in general, a shorter repolarization results in a shorter QT interval $(1,24)$. Therefore, a smaller QT index in REM sleep is consistent with an increase in sympathetic discharge. On the other hand, vagal stimulation results in a prolongation of the refractory period and prolongation of ventricular repolarization $(15,22)$. However, previous data have shown no dramatic effect of acetylcholine on the rate of repolarization of the mammalian ventricle (8). Thus, we believe that the small sleep staterelated difference in the duration of the QT interval is mainly caused by an increased sympathetic activity in REM sleep, leading to a shortening of the QT interval.

Several investigators have postulated that the etiology of SIDS might be related to an abnormal prolongation of the QT interval in sleep $(7,17,20)$, especially REM sleep $(20)$. The present study demonstrates that, in normal infants, the QT index is smaller in REM than in quiet sleep. Thus, it provides data for the evaluation of the sleep state-related differences in the QT interval in the infant at risk for SIDS.

\section{REFERENCES AND NOTES}

1. Abildskov, J. A.: Adrenergic effects on the QT interval of the electrocardiogram. Am. Heart J., 92: 210 (1976).

2. Alimurung, M. M., Joseph, L. G., Craige, E., and M Massell, B. F.: The QT interval in normal infants and children. Circulation, $l: 1329$ (1950).

3. Anders, T., Emde, R., and Parmelee, A.: A manual of standardized terminology, techniques and criteria for scoring states of sleep and wakefulness in infants 
(UCLA Brain Information Service, BRI Publications Offices, Los Angeles, 1971).

4. Baust, W., and Bohnert, B.: The regulation of heart rate during sleep. Exp. Brain Res., 7: 169 (1969).

5. Baust, W., Weidinger, H., and Kirchner, F.: Sympathetic activity during natural sleep and arousal. Arch. Ital. Biol., 106: 379 (1968).

6. Bazett, $H$. C.: An analysis of the time relations of electrocardiograms. Heart, 7: 353 (1920).

7. Ferrer, P. L., and Jesse, M. J.: Prolonged QT index in "near-miss" sudden death in infancy. Clin. Res., 25: 64A (1977).

8. Fisch, C., Knoebel, S. B., and Feigenbaum, H.: The effect of acetylcholine and potassium on repolarization of the heart. J. Clin. Invest., 43: 1769 (1964).

9. Fisher, R. A., and Yates, F.: Statistical Tables (Hafner, Darien, 1970).

10. Giotti, A., Ledda, F., and Mannaioni, P. F.: Effects of noradrenaline and isoprenaline, in combination with $\alpha$ and $\beta$-receptor blocking substances, on the action potential of cardiac purkinje fibers. J. Physiol. (Lond), 229: 29 (1973).

11. Han, J., de Jalon, P. G., and Moe, G. K.: Adrenergic effects of ventricular vulnerability. Circ. Res., 14: 516 (1964).

12. Harper, R. M., Kelly, D. S., Walter, D. O., and Hoppenbrouwers, T.: Cardiac waveform alterations during sleep in the infant. Psychophysiology, 13: 318, (1976).

13. Iwamura, Y., Uchino, Y., and Kidokoro, Y.: Blood pressure and heart rate changes during para-sleep in vagotomized and atropinized cats. Brain Res., 7: 182 (1968).

14. Jervell, A., and Lange-Nielson, F.: Congenital deaf-mutism, functional heart disease with prolongation of the QT interval, and sudden death. Am Heart J., 57: 59 (1957).

15. Kolman, B. S., Verrier, R. L., and Lown, B.: The effect of vagus nerve stimulation upon excitability of the canine ventricle; role of sympathetic-parasympathetic interactions. Am. J. Cardiol., 37: 1042 (1976).

16. Leistner, H. L., Haddad, G. G., Epstein, R. A., Epstein, M. A. F., Mazza, N. M., and Mellins, R. B.: Heart rate and heart rate variability in normal and aborted

Copyright $\odot 1979$ International Pediatric Research Foundation, Inc $0031-3998 / 79 / 1302-0139 \$ 02.00 / 0$
SIDS infants during sleep. Pediatr. Res. 12: 528 (1978).

17. Maron, B. J., Goldstein, R. E., Clark, G. E., Fisher, R. S., and Epstein, S. E.: Potential role of QT interval prolongation in sudden infant death syndrome. Circulation, 54: 423 (1976).

18. Nomenclature and criteria for diagnosis of diseases of the heart and great vessels. The criteria committee of the N.Y.H.A., 7th edition (Little, Brown and Company, Boston, 1973).

19. Prechtl, H. F. R.: Polygraphic studies of the full-term newborn: II. Computer analysis of recorded data. In: R. McKeith and M. Bax: Studies in Infancy (Clinics in Developmental Medicine), Vol. 27, p. 22 (Heinemann Medical, London, 1968).

20. Schwartz, P. J.: Cardiac sympathetic innervation and the sudden infant death syndrome. A possible pathogenetic link. Am. J. Med., 60: 167 (1976).

21. Schwartz, P. J., Periti, M., and Malliani, A.: The long Q-T syndrome. Am. Heart J., 89: 378 (1975).

22. Schwartz, P. J., Verrier, R. L., and Lown, B.: Effect of stelletomy and vagotomy of ventricular refractoriness in dogs. Circ. Res., 40:536 (1977).

23. Shoenwald, R. D., and Isaacs, V. E.: Q-T corrected for heart rate: A new approach and its application. Arch. Int. Pharmacodyn., 21l: 34 (1974).

24. Vincent, G. M., Abildskov, J. A., Burgess, M. J.: Q-T interval syndromes. Prog. Cardiovasc. Dis., 16: 523 (1974).

25. Ziegler, R. F.: Ventricular activation. In: Electrocardiographic Studies in Normal Infants and Children, p. 46 (Charles C Thomas, Springfield, 1951).

26. The authors are grateful to Dr. Brian F. Hoffman for critical review of the manuscript and Mr. Henry Puchalski for technical assistance.

27. This research was supported by National Institutes of Health Grants HD 08297 and HL 06012 and Contract HD 52856.

28. Requests for reprints should be addressed to: G. G. Haddad, M.D., Pediatric Pulmonary Division, Columbia-Presbyterian Medical Center, 622 West 168th Street, New York, NY 10032 (USA).

29. Received for publication January 20, 1978

30. Accepted for publication March 21, 1978. 